ISSN 0258-7122 (Print), 2408-8293 (Online)

Bangladesh J. Agril. Res. 43(3): 383-393, September 2018

\title{
PERFORMANCE OF HYBRID LINES OF POINTED GOURD (Trichosanthes dioica Roxb) FOR YIELD AND YIELD ATTRIBUTES
}

\author{
N. ARA ${ }^{1}$, M. MONIRUZZAMAN ${ }^{2}$, K. S. RAHMAN ${ }^{3}$ \\ M. MONIRUZZAMAN ${ }^{4}$ AND R. SULTANA ${ }^{5}$
}

\begin{abstract}
A field experiment was conducted at the Regional Agricultural Research Station, Ishurdi, Pabna during the growing season of 2013-14 with eighteen hybrid lines of pointed gourd and BARI Hybrid Patal -1 as check to observe their performances for yield, yield attributes and other morphological characters. The experiment was laid out in randomized complete block design with three replications. The maximum vine length $(242.00 \mathrm{~cm})$ and nodes/plant $(18.30)$ was recorded from $\mathrm{PG} 018 \times \mathrm{M}_{2}$. Shoots/plant ranged from $12.50\left(\mathrm{PG} 027 \times \mathrm{M}_{2}\right)$ to $4.66\left(\mathrm{PG} 008 \mathrm{M}_{1}\right)$. The maximum number of fruits/plant (160.00) was obtained from $\mathrm{PG} 009 \times \mathrm{M}_{2}$ followed by $\mathrm{PG} 012 \times \mathrm{M}_{1}$ (154.66). Individual fruit weight was recorded highest $(50.10 \mathrm{~g})$ in $\mathrm{PG} 027 \times \mathrm{M}_{2}$, which was very close to PG008 $\times \mathrm{M}_{2}$ (48.00) and $\mathrm{PG} 018 \times \mathrm{M}_{2}(47.00 \mathrm{~g})$. Weight of fruits/plant ranged from $\mathrm{PG} 009 \times \mathrm{M}_{2}(6.86 \mathrm{~kg})$ to $\mathrm{PG} 022 \times \mathrm{M}_{1}(3.01 \mathrm{~kg})$. The highest pulp weight was recorded in $\mathrm{PG} 027 \times \mathrm{M}_{2}(44.20 \mathrm{~g})$ which was statistically similar to $\mathrm{PG} 008 \times \mathrm{M}_{2}$ $(42.20 \mathrm{~g})$. Three different leaf colour (light green, green and deep green), two types of leaf tip (pointed and blunt) and four types of leaf margin (slightly serrated, serrated, entire and undulated) were found among the hybrid lines. Four fruit colour (whitish, light green, green and dark green), four fruit stripes (no stripe, white, green white and light green) and three types of fruit curvature (slightly curved, curved and straight) were observed in different lines. The line PG009 $\times \mathrm{M}_{2}$ showed better performance in respect of fruits/plant and weight of fruits/plant and thus gave the highest yield (45.74 t/ha). The lines PG008 $\times \mathrm{M}_{2}$, $\mathrm{PG} 007 \times \mathrm{M}_{2}, \mathrm{PG} 017 \times \mathrm{M}_{2}, \mathrm{PG} 027 \times \mathrm{M}_{2}$ and $\mathrm{PG} 014 \times \mathrm{M}_{1}$ also produced better yield (39.23 - $35.58 \mathrm{t} / \mathrm{ha}$ ). Therefore, the lines PG014 $\times \mathrm{M}_{1}, \mathrm{PG} 007 \times \mathrm{M}_{2}, \mathrm{PG} 008 \times \mathrm{M}_{2}$, $\mathrm{PG} 009 \times \mathrm{M}_{2}$ and $\mathrm{PG} 017 \times \mathrm{M}_{2}$ should be subjected for further evaluation to release as variety.
\end{abstract}

Keywords: Hybrid lines, pointed gourd, Trichosanthes dioica and yield.

\section{Introduction}

Pointed gourd (Trichosanthes dioica Roxb.) locally known as 'Patal' is a popular cucurbitaceous vegetable in Bangladesh. The Bengal and Assam region of India

${ }^{1}$ Chief Scientific Officer, Plant Physiology Division, Bangladesh Agricultural Research Institute (BARI), Gazipur, ${ }^{2}$ Principal Scientific Officer, Plant Physiology Section, HRC, BARI, Gazipur, ${ }^{3}$ Scientific Officer, ASICT Division, BARI, Gazipur, ${ }^{4}$ Scientific Officer, Plant Physiology Section, HRC, BARI, Gazipur, ${ }^{5}$ Principal Scientific Officer, Training \& Communication Wing, BARI, Gazipur, Bangladesh. 
are the primary centre of its origion (Singh et al., 1992). It is cultivated almost in all districts of Bangladesh, especially in Rajshahi, Pabna, Jessore, Bogra, Rangpur and Kushtia in summer and rainy season (Rashid, 1993). It is morphologically distinct from the other cucurbitaceous species due to its well established dioecious vegetative means of propagation (Awal et al., 2005). In Bangladesh, pointed gourd is cultivated in an area of around 10006.5 ha with total production of 84096 metric tons and national average yield is 8.40 t/ha during 2014-15 (Anon., 2016). It is seen that average yield of pointed gourd $(8.40 \mathrm{t} / \mathrm{ha})$ is low compared to other countries like India (FAO, 1981) due to lack of enough high yielding varieties.

Besides carbohydrates, it provides considerable amounts of minerals. $100 \mathrm{~g}$ edible portion of pointed gourd contains $83.0 \mathrm{mg} \mathrm{Cu}, 17.0 \mathrm{mg} \mathrm{S}, 9.0 \mathrm{mg} \mathrm{Mg}$ and $2.6 \mathrm{mg} \mathrm{Na}$ (Singh, 1989). Scarcity of vegetables prevails in the market at the end of winter and beginning of summer seasons. During the months of May to October, only a few vegetables are available in the market and the quantity is very low against the demand. Pointed gourd becomes available in the market from March to the end of October. At this juncture, pointed gourd can fulfill the demand of vegetables to some extent.

There are three released pointed gourd varieties, two open pollinated and one hybrid in Bangladesh, which are not enough for the farmers. Farmers need more number of high yielding varieties including hybrid for cultivation. Pointed gourd is dioecious in nature and propagated vegetatively through root suckers and vines. Therefore, development and maintenance of hybrid is easier than other crops. Some hybrid lines have been developed at the Regional Agricultural Research Station, Ishurdi, Pabna. Among those lines, one line $\left(\mathrm{PG} 027 \times \mathrm{M}_{2}\right)$ has been released as BARI Hybrid Patal-1 after evaluation and other lines need to be evaluated. The study was, therefore, undertaken to evaluate different hybrid lines for selection of superior hybrid varieties of pointed gourd.

\section{Materials and Method}

The experiment was conducted at the Regional Agricultural Research Station of Bangladesh Agricultural Research Institute (BARI), Ishurdi, Pabna, during the growing period of October 2013 to October 2014. The experimental site was at $24.03^{\circ} \mathrm{N}$ Latitude and $89.05^{\circ} \mathrm{E}$ Longitude with an elevation of $16 \mathrm{~m}$ above from the sea level. The soil of the experimental field was clay loam with $\mathrm{pH}$ value of 8.5 belonging to the High Ganges River Floodplain under AEZ -11 (BARC, 2005). The crop received $1175 \mathrm{~mm}$ annual rainfall during the whole growing season. The treatment consisted of 18 hybrid lines of pointed gourd viz., PG002 $\times M_{1}, \quad P G 003 \times M_{1}, \quad P G 004 \times M_{1}, \quad P G 005 \times M_{1}, \quad P G 006 \times M_{1}, \quad P G 008 \times M_{1}$, $P G 012 \times M_{1}, \quad P G 014 \times M_{1}, \quad P G 022 \times M_{1}, \quad P G 002 \times M_{2}, \quad P G 005 \times M_{2}, \quad P G 006 \times M_{2}$, $\mathrm{PG} 007 \times \mathrm{M}_{2}, \mathrm{PG} 008 \times \mathrm{M}_{2}, \mathrm{PG} 009 \times \mathrm{M}_{2}, \mathrm{PG} 017 \times \mathrm{M}_{2}, \mathrm{PG} 018 \times \mathrm{M}_{2}$ and $\mathrm{PG} 025 \times \mathrm{M}_{2}$ 
with BARI Hybrid Patal-1 as check. The fresh vines of male lines were planted on 10 October 2013 in some plots where female plants were not planted. The male and female population was maintained at 1:10 ratio. The hybrid lines of pointed gourd were evaluated in randomized complete block design with three replications. The land was ploughed well with the help of tractor followed by harrowing. Unit plot size was $4.5 \mathrm{~m} \times 1.0 \mathrm{~m}$. The fresh vines of different female lines were planted on 10 October 2013 maintaining the spacing of $1.5 \mathrm{~m} \times 1.0 \mathrm{~m}$. Watering was done in the morning for establishment of the vines. Manure and fertilizers were applied @ 10 tons of cowdung, $500 \mathrm{~kg}$ of Urea, $360 \mathrm{~kg}$ of Triple Super Phosphate, $330 \mathrm{~kg}$ of Muriate of Potash and $200 \mathrm{~kg}$ of Gypsum per hectare (Khan et al., 2007). Full doses of cowdung, Triple Super Phosphate and gypsum were applied as basal during pit preparation. Urea and Muriate of Potash were top dressed in three equal installments at 20,60 and 90 days after emergence (Khan et al., 2007). Irrigation and other intercultural operations were done as and when necessary. Bamboo branches were used to support the plants and the plants were allowed to trail on the trellises made of bamboo slices. Vines emerged out from the plants near the soil level were pruned during the growing period. Fruits were harvested regularly when attained maturity or immediately before hardness of seeds (about 12 days after anthesis). Harvesting of fruit was started on 23 March 2014 and continued upto 28 October 2014 in different lines. Data on various morphological and yield characters were recorded from three plants of each plot following Plant Genetic Resources Descriptor (IBPGR, 1983). Fresh fruits were harvested from the field treatment wise in the month of July 2014 and brought them to the laboratory. Then ten fruits were randomly selected and kept treatment wise in white polybag $(25 \mathrm{~cm} \times 20 \mathrm{~cm})$ having some holes in ambient condition. Days counted from first day (on harvest day) to the day at which fruits became shriveled, pale in colour and unsuitable for consumption were considered for shelf life.

Collected data were subjected to statistical analysis through MSTAT C Software and means were compared by DMRT at 5\% level of probability.

\section{Results and Discussion}

\section{A) Days to $1^{\text {st }}$ female flowering, vine length, number of node and shoot}

The pointed gourd lines showed wide variation in days to first female flowering (Table 1). Appearance of first female flower ranged from $151\left(\mathrm{PG} 005 \times \mathrm{M}_{2}\right)$ to $170\left(\mathrm{PG} 008 \times \mathrm{M}_{2}\right)$ days. Variation in flowing period in pointed gourd was also observed by Shanmugavelu (1989). The line PG018 $\times \mathrm{M}_{2}$ produced the highest vine length $(242.00 \mathrm{~cm})$ which was statistically similar to $\mathrm{PG} 025 \times \mathrm{M}_{2}(234.00$ $\mathrm{cm})$ and $\mathrm{PG} 027 \times \mathrm{M}_{2}(233.00 \mathrm{~cm})$. The line $\mathrm{PG} 007 \times \mathrm{M}_{2}$ produced the lowest vine length $(90.00 \mathrm{~cm})$. Significant variation in vine length of pointed gourd was also reported by Kumar et al. (1995) and Kabir (2007). Kumar et al. (1995) found the vine length ranging from $180.00 \mathrm{~cm}$ to $267 \mathrm{~cm}$; whereas, Kabir (2007) found 
vine length with a range of $95 \mathrm{~cm}$ to $470 \mathrm{~cm}$. The maximum number of nodes/plant was found in PG018 $\times \mathrm{M}_{2}$ (18.30) followed by $\mathrm{PG} 006 \times \mathrm{M}_{1}(17.30)$, $\mathrm{PG} 25 \times \mathrm{M}_{2}$ (16.60), $\mathrm{PG} 027 \times \mathrm{M}_{2}$ (16.33), $\mathrm{PG} 017 \times \mathrm{M}_{2}$ (16.00), $\mathrm{PG} 002 \times \mathrm{M}_{1}(15.00)$, $\mathrm{PG}_{012} \times \mathrm{M}_{1}$ (15.00), PG008 $\times \mathrm{M}_{2}$ (14.30), PG002 $\times \mathrm{M}_{2}(14.00)$ and $\mathrm{PG} 007 \times \mathrm{M}_{2}$ (14.00). Number of nodes/vine increased with the increasing vine length. Singh and Prasad (1989) also observed a wide range of variation for number of nodes/vine in pointed gourd. Number of shoots/plant ranged from 4.66 to 12.50 (Table 1). BARI Hybrid Patal 1 produced the maximum shoots/plant (12.50) which was identical to $\mathrm{PG} 006 \times \mathrm{M}_{1}(12.00), \mathrm{PG} 012 \times \mathrm{M}_{1}(11.00), \mathrm{PG} 003 \times \mathrm{M}_{1}$ (10.66), $\mathrm{PG} 002 \times \mathrm{M}_{1}(10.33)$ and $\mathrm{PG} 014 \times \mathrm{M}_{1}$ (10.33), while the minimum in $\mathrm{PG} 008 \times \mathrm{M}_{1}$ (4.66).

Table 1. Days to first female flowering, vine length, number of nodes per plant and shoots per plant of nineteen hybrid pointed gourd lines

\begin{tabular}{|c|c|c|c|c|}
\hline Hybrid lines & $\begin{array}{l}\text { Days to } 1^{\text {st }} \\
\text { female } \\
\text { flowering }\end{array}$ & $\begin{array}{l}\text { Length of vine } \\
\qquad(\mathrm{cm})\end{array}$ & $\begin{array}{c}\text { Nodes/plant } \\
\text { (no.) }\end{array}$ & $\begin{array}{c}\text { Shoots/plant } \\
\text { (no.) }\end{array}$ \\
\hline $\mathrm{PG} 002 \times \mathrm{M}_{1}$ & 164def & $172.00 \mathrm{c}$ & $15.00 \mathrm{a}-\mathrm{f}$ & $10.33 \mathrm{ab}$ \\
\hline $\mathrm{PG} 003 \times \mathrm{M}_{1}$ & 164def & 114.00f-h & $10.00 \mathrm{~g}-\mathrm{i}$ & $10.66 \mathrm{ab}$ \\
\hline $\mathrm{PG} 004 \times \mathrm{M}_{1}$ & $168 \mathrm{abc}$ & $183.00 \mathrm{j}$ & $8.30 \mathrm{~h}-\mathrm{i}$ & $8.00 \mathrm{~d}$ \\
\hline $\mathrm{PG} 005 \times \mathrm{M}_{1}$ & $161 \mathrm{f}$ & $109.00 \mathrm{gh}$ & $15.30 \mathrm{a}-\mathrm{e}$ & $9.66 b c$ \\
\hline $\mathrm{PG} 006 \times \mathrm{M}_{1}$ & $164 \mathrm{def}$ & $218.00 \mathrm{~b}$ & $17.30 \mathrm{ab}$ & $12.00 \mathrm{a}$ \\
\hline $\mathrm{PG} 008 \times \mathrm{M}_{1}$ & $153 \mathrm{gh}$ & $113.00 \mathrm{f}-\mathrm{h}$ & $11.33 \mathrm{~d}-\mathrm{i}$ & $4.66 f$ \\
\hline PG012×M & $156 \mathrm{~g}$ & $214.00 \mathrm{~b}$ & $15.00 \mathrm{a}-\mathrm{f}$ & $11.00 \mathrm{ab}$ \\
\hline $\mathrm{PG} 014 \times \mathrm{M}_{1}$ & $166 \mathrm{bcd}$ & 101.00hi & $10.33 \mathrm{f}-\mathrm{i}$ & $10.33 \mathrm{ab}$ \\
\hline $\mathrm{PG} 022 \times \mathrm{M}_{1}$ & 166bcd & $139.00 \mathrm{~d}$ & $11.00 \mathrm{e}-\mathrm{i}$ & $6.33 \mathrm{~d}-\mathrm{f}$ \\
\hline $\mathrm{PG} 002 \times \mathrm{M}_{2}$ & $162 \mathrm{ef}$ & $143.06 \mathrm{~d}$ & $14.00 \mathrm{a}-\mathrm{g}$ & 7.30de \\
\hline $\mathrm{PG} 005 \times \mathrm{M}_{2}$ & $151 \mathrm{~h}$ & $121.00 \mathrm{fg}$ & $12.60 \mathrm{~b}-\mathrm{h}$ & $8.00 \mathrm{~cd}$ \\
\hline $\mathrm{PG} 006 \times \mathrm{M}_{2}$ & $163 \mathrm{ef}$ & $92.00 \mathrm{ij}$ & 7.30i & $5.66 \mathrm{ef}$ \\
\hline $\mathrm{PG} 007 \times \mathrm{M}_{2}$ & $166 \mathrm{bcd}$ & $90.00 \mathrm{ij}$ & $14.00 \mathrm{a}-\mathrm{g}$ & $5.60 \mathrm{ef}$ \\
\hline $\mathrm{PG} 008 \times \mathrm{M}_{2}$ & $170 \mathrm{a}$ & $136.00 \mathrm{e}$ & $14.30 \mathrm{a}-\mathrm{g}$ & $7.33 \mathrm{de}$ \\
\hline $\mathrm{PG} 009 \times \mathrm{M}_{2}$ & $166 \mathrm{bcd}$ & $125.00 \mathrm{ef}$ & $11.6 \mathrm{c}-\mathrm{i}$ & $8.00 \mathrm{~cd}$ \\
\hline $\mathrm{PG} 017 \times \mathrm{M}_{2}$ & $169 \mathrm{ab}$ & $219.00 b$ & $16.00 \mathrm{a}-\mathrm{d}$ & 7.00de \\
\hline $\mathrm{PG} 018 \times \mathrm{M}_{2}$ & $163 \mathrm{ef}$ & $242.00 \mathrm{a}$ & $18.30 \mathrm{a}$ & $8.00 \mathrm{~cd}$ \\
\hline $\mathrm{PG} 025 \times \mathrm{M}_{2}$ & $165 \mathrm{cde}$ & $234.30 \mathrm{a}$ & $16.60 \mathrm{ab}$ & 7.33de \\
\hline BARI Hybrid Patal -1 & $157 \mathrm{~g}$ & $233.00 \mathrm{a}$ & $16.33 \mathrm{a}-\mathrm{c}$ & $12.50 \mathrm{a}$ \\
\hline $\mathrm{CV}(\%)_{-}$ & 6.70 & 4.95 & 18.17 & 11.45 \\
\hline
\end{tabular}

Means with uncommon letter(s) in a column are significantly different at 5\% level by DMRT. 
Table 2. Number of fruits per plant, fruit size, individual fruit weight, weight of fruits per plant of nineteen hybrid pointed gourd lines

\begin{tabular}{|c|c|c|c|c|c|c|}
\hline Hybrid lines & $\begin{array}{l}\text { Days to } \\
1^{\text {st }} \text { fruit } \\
\text { harvest }\end{array}$ & $\begin{array}{l}\text { Fruits/plant } \\
\text { (no.) }\end{array}$ & $\begin{array}{l}\text { Individual } \\
\text { fruit } \\
\text { weight } \\
(\mathrm{g})\end{array}$ & $\begin{array}{l}\text { Length } \\
\text { of fruit } \\
(\mathrm{cm})\end{array}$ & $\begin{array}{l}\text { Width } \\
\text { of fruit } \\
(\mathrm{cm})\end{array}$ & $\begin{array}{l}\text { Weight of } \\
\text { fruits/plant } \\
\quad(\mathrm{kg})\end{array}$ \\
\hline $\mathrm{PG} 002 \times \mathrm{M}_{1}$ & $176 b c$ & $107.33 \mathrm{ij}$ & $40.24 d-f$ & $10.00 \mathrm{c}$ & $3.30 \mathrm{ef}$ & $4.32 \mathrm{~d}$ \\
\hline $\mathrm{PG} 003 \times \mathrm{M}_{1}$ & $175 c$ & $98.00 \mathrm{k}$ & $45.61 \mathrm{a}-\mathrm{d}$ & $11.56 \mathrm{~b}$ & $3.53 \mathrm{~d}-\mathrm{f}$ & $4.47 \mathrm{~cd}$ \\
\hline $\mathrm{PG} 004 \times \mathrm{M}_{1}$ & $179 \mathrm{abc}$ & $115.66 \mathrm{e}$ & $44.49 \mathrm{a}-\mathrm{d}$ & $11.50 \mathrm{~b}$ & $3.50 \mathrm{~d}-\mathrm{f}$ & $5.14 \mathrm{c}$ \\
\hline $\mathrm{PG} 005 \times \mathrm{M}_{1}$ & $175 \mathrm{c}$ & $114.00 \mathrm{~g}-\mathrm{i}$ & $38.00 \mathrm{e}-\mathrm{h}$ & $10.06 \mathrm{c}$ & $3.60 \mathrm{c}-\mathrm{f}$ & $4.38 \mathrm{~d}$ \\
\hline $\mathrm{PG} 006 \times \mathrm{M}_{1}$ & $175 \mathrm{c}$ & $121.66 \mathrm{~d}-\mathrm{f}$ & $37.00 \mathrm{e}-\mathrm{h}$ & $8.36 f g$ & $3.53 \mathrm{~d}-\mathrm{f}$ & $5.25 \mathrm{c}$ \\
\hline $\mathrm{PG} 008 \times \mathrm{M}_{1}$ & $176 \mathrm{c}$ & $117.66 \mathrm{e}-\mathrm{g}$ & 43.66b-e & $9.83 \mathrm{~cd}$ & $3.20 \mathrm{f}$ & $4.38 \mathrm{~d}$ \\
\hline $\mathrm{PG} 012 \times \mathrm{M}_{1}$ & $165 c$ & $154.66 \mathrm{a}$ & $34.14 \mathrm{gh}$ & $9.40 \mathrm{c}-\mathrm{e}$ & $3.63 \mathrm{c}-\mathrm{e}$ & $5.26 \mathrm{c}$ \\
\hline $\mathrm{PG} 014 \times \mathrm{M}_{1}$ & $168 \mathrm{c}$ & $117.33 \mathrm{e}-\mathrm{g}$ & $45.62 a-d$ & $11.33 b$ & $3.70 \mathrm{~b}-\mathrm{e}$ & $5.33 c$ \\
\hline $\mathrm{PG} 022 \times \mathrm{M}_{1}$ & $178 \mathrm{abc}$ & $135.00 \mathrm{~b}$ & $22.92 \mathrm{i}$ & $7.83 \mathrm{~g}$ & $3.60 c-f$ & $3.01 \mathrm{f}$ \\
\hline $\mathrm{PG} 002 \times \mathrm{M}_{2}$ & $178 \mathrm{abc}$ & $107.35 \mathrm{ij}$ & $32.40 \mathrm{~h}$ & $9.50 \mathrm{c}-\mathrm{e}$ & $3.30 \mathrm{ef}$ & $3.46 \mathrm{e}$ \\
\hline $\mathrm{PG} 005 \times \mathrm{M}_{2}$ & $176 b c$ & 115.00f-h & $44.40 \mathrm{a}-\mathrm{d}$ & $9.53 \mathrm{c}-\mathrm{e}$ & $3.46 \mathrm{~d}-\mathrm{f}$ & $5.10 \mathrm{c}$ \\
\hline $\mathrm{PG} 006 \times \mathrm{M}_{2}$ & $167 d$ & $125.66 \mathrm{~cd}$ & $39.60 \mathrm{~d}-\mathrm{g}$ & $9.16 \mathrm{de}$ & $3.53 \mathrm{~d}-\mathrm{f}$ & $4.95 \mathrm{c}$ \\
\hline $\mathrm{PG} 007 \times \mathrm{M}_{2}$ & $177 \mathrm{bc}$ & $129.00 \mathrm{bc}$ & $41.27 \mathrm{c}-\mathrm{f}$ & $8.90 \mathrm{ef}$ & $3.66 \mathrm{c}-\mathrm{e}$ & $5.32 \mathrm{c}$ \\
\hline $\mathrm{PG} 008 \times \mathrm{M}_{2}$ & $178 \mathrm{abc}$ & $120.00 \mathrm{~d}-\mathrm{g}$ & $48.00 \mathrm{ab}$ & $12.67 \mathrm{a}$ & $3.96 \mathrm{a}-\mathrm{c}$ & $5.88 \mathrm{~b}$ \\
\hline $\mathrm{PG} 009 \times \mathrm{M}_{2}$ & $182 \mathrm{a}$ & $160.00 \mathrm{a}$ & $43.00 \mathrm{~b}-\mathrm{f}$ & $10.16 \mathrm{c}$ & $3.76 a-d$ & $6.86 a$ \\
\hline PG017× $\mathrm{M}_{2}$ & $178 \mathrm{abc}$ & $129.00 \mathrm{bc}$ & $42.00 \mathrm{~b}-\mathrm{f}$ & $9.56 c-e$ & $4.10 \mathrm{ab}$ & $5.43 c$ \\
\hline $\mathrm{PG} 018 \times \mathrm{M}_{2}$ & $181 \mathrm{ab}$ & $109.66 \mathrm{~h}-\mathrm{j}$ & $47.00 \mathrm{a}-\mathrm{c}$ & $9.93 \mathrm{~cd}$ & $4.16 \mathrm{a}$ & $5.15 c$ \\
\hline $\mathrm{PG} 025 \times \mathrm{M}_{2}$ & $175 \mathrm{c}$ & $123.00 \mathrm{c}-\mathrm{e}$ & $41.24 c-f$ & $9.13 \mathrm{de}$ & $3.56 c-f$ & $5.07 \mathrm{c}$ \\
\hline $\begin{array}{c}\text { BARI Hybrid Patal } \\
-1\end{array}$ & $178 \mathrm{abc}$ & $104.00 \mathrm{ij}$ & $50.10 \mathrm{a}$ & $13.23 \mathrm{a}$ & $4.10 \mathrm{ab}$ & $5.39 \mathrm{c}$ \\
\hline $\mathrm{CV}(\%)$ & 5.29 & 3.24 & 7.76 & 4.19 & 6.03 & 5.06 \\
\hline
\end{tabular}

Means with uncommon letter (s) in a column are significantly different at $5 \%$ level by DMRT.

\section{B) Days to $1^{\text {st }}$ fruit harvest, number of fruits/plant, individual fruit weight, fruit length and width and weight of fruits/plant}

Days to $1^{\text {st }}$ fruit harvest, fruits/plant, fruit length, fruit width, individual fruit weight and weight of fruits/plant showed significant variation in hybrid pointed gourd lines (Table 2). Days to $1^{\text {st }}$ fruit harvest ranged from 165 to 182 days. Maximum days were required to $1^{\text {st }}$ fruit harvest of $\mathrm{PG} 009 \times \mathrm{M}_{2}$ (182 days)

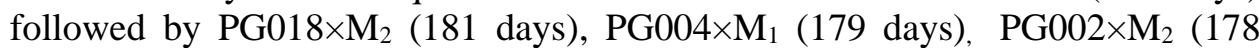


days), PG017×M (178 days), BARI Hybrid Patal 1 (178 days) and PG022 $\times \mathrm{M}_{1}$ (178 days), and the minimum time required for $\mathrm{PG} 012 \times \mathrm{M}_{1}(165$ days $)$. The number of fruits/plant ranged from 98.00 to 160.00 . Maximum number of fruits /plant was recorded from PG009 $\times \mathrm{M}_{2}$ (160.00) followed by PG012 $\times \mathrm{M}_{1}(154.66)$ and the minimum from $\mathrm{PG} 003 \times \mathrm{M}_{1}$ (98.00). The variation in number of fruits/plant among the pointed gourd genotypes was also reported by Prasad and Singh (1990) and Alam et al. (2008). Alam et al. (2008) found the fruit number/plant with a range of 13.67 to 180.67 . Individual fruit weight varied significantly among the lines and it ranged from $22.92 \mathrm{~g}$ to $50.10 \mathrm{~g}$. The largest fruit was obtained from BARI Hybrid Patal-1 $(50.10 \mathrm{~g})$ followed by PG008 $\times \mathrm{M}_{2}$ $(48.00 \mathrm{~g}), \mathrm{PG} 018 \times \mathrm{M}_{2}(47.00 \mathrm{~g}), \mathrm{PG} 003 \times \mathrm{M}_{1}(45.61 \mathrm{~g}), \mathrm{PG} 014 \times \mathrm{M}_{1}(45.62 \mathrm{~g})$, PG004 $\times \mathrm{M}_{1}(44.49 \mathrm{~g})$ and PG005 $\times \mathrm{M}_{2}(44.40 \mathrm{~g})$; whereas, the smallest fruit was produced by $\mathrm{PG} 022 \times \mathrm{M}_{1}(22.92 \mathrm{~g})$. The fruit length ranged from 7.83 to 13.23 $\mathrm{cm}$. The highest fruit length was in BARI Hybrid Patal-1 $(13.23 \mathrm{~cm})$ which was closely followed by PG008 $\times \mathrm{M}_{2}(12.67 \mathrm{~cm})$ and the smallest from PG022 $\times \mathrm{M}_{1}$ $(7.83 \mathrm{~cm})$. These results are almost similar to Khan et al. (2007) and Alam et al. (2008). The widest fruit was produced by $\mathrm{PG} 018 \times \mathrm{M}_{2}(4.16 \mathrm{~cm})$ followed by PG017 $\times \mathrm{M}_{2}$ and Bari hybrid Patal $1(4.10 \mathrm{~cm})$ but the narrowest in PG008 $\times \mathrm{M}_{1}$ $(3.20 \mathrm{~cm}$ ). Weight of fruits/plant varied significantly among the lines (Table 2). The maximum weight of fruits $/$ plant was recorded in $\mathrm{PG} 009 \times \mathrm{M}_{2}(6.86 \mathrm{~kg})$ which differed significantly from others but the minimum was found in PG022 $\times \mathrm{M}_{1}$ $(3.01 \mathrm{~kg})$. The better fruit weight $/$ plant was also recorded in $\mathrm{PG} 008 \times \mathrm{M}_{2}(5.88 \mathrm{~kg})$, $\mathrm{PG} 027 \times \mathrm{M}_{2}(5.39 \mathrm{~kg})$ and $\mathrm{PG} 014 \times \mathrm{M}_{1}(5.33 \mathrm{~kg})$. Similar results were also reported by Singh et al. (1985), Alam et al. (2008) and Khan et al. (2007).

\section{C) Pulp weight, number of seeds/fruit, weight of seeds/fruit, duration of fruit harvest, fruit yield and shelf life}

Pulp weight/fruit, number of seeds/fruit, weight of seeds/fruit, duration of fruit harvest, fruit yield and shelf life are presented in Table 3. Pulp weight in the lines ranged from $17.20 \mathrm{~g}$ to $44.20 \mathrm{~g}$. The maximum pulp weight was obtained from the fruit of BARI Hybrid Patal 1 (44.20 g) followed by PG008 $\times \mathrm{M}_{2}$ (42.20 g). On the other hand, the minimum pulp weight was produced by PG022 $\times \mathrm{M}_{1}(17.20 \mathrm{~g})$. These results are in conformity with the findings of Kabir (2007), Khan et al. (2007) and Alam et al. (2008). Number of seeds/fruit ranged from 16.66 to 27.33. The maximum number of seeds/fruit was recorded in $\mathrm{PG} 014 \times \mathrm{M}_{1}$ (27.33) closely followed by PG018 $\times \mathrm{M}_{2}, \mathrm{PG} 007 \times \mathrm{M}_{2}, \mathrm{PG} 025 \times \mathrm{M}_{2}, \mathrm{PG} 008 \times \mathrm{M}_{2}$, and PG012 $\times \mathrm{M}_{1}$, while the minimum in $\mathrm{PG} 005 \times \mathrm{M}_{1}$ (16.66). This corroborates the findings of other investigators (Khan et al., 2007; Alam et al., 2008; Prasad and Singh, 1990). The maximum weight of seeds/fruits was found in PG018 $\times \mathrm{M}_{2}(7.20 \mathrm{~g})$ which was closely followed by PG006 $\times \mathrm{M}_{2}(7.10 \mathrm{~g})$ while the lowest in PG005 $\times \mathrm{M}_{1}(4.13 \mathrm{~g})$. Significant variation was also found in the lines with regard to duration of fruit harvest which ranged from 212 days to 223 days. The maximum harvest duration 
was found in PG017 $\times \mathrm{M}_{2}$ (223 days) followed by PG009 $\times \mathrm{M}_{2}$ (222 days) and the minimum duration was recorded in $\mathrm{PG} 002 \times \mathrm{M}_{2}\left(212\right.$ days) and $\mathrm{PG} 005 \times \mathrm{M}_{2}(212$ days). Yield of pointed gourd significantly varied among the lines (Table 3 ). The maximum yield was obtained from $\mathrm{PG} 009 \times \mathrm{M}_{2}(45.74 \mathrm{t} / \mathrm{ha})$ followed by PG008 $\times \mathrm{M}_{2}$ (39.23 t/ha), BARI Hybrid Patal-1 (36.85 t/ha), PG017 $\times \mathrm{M}_{2}$ (36.19 $\mathrm{t} / \mathrm{ha}$ ) and $\mathrm{PG} 027 \times \mathrm{M}_{2}(35.94 \mathrm{t} / \mathrm{ha})$ and the minimum was recorded in $\mathrm{PG} 0022 \times \mathrm{M}_{1}$ (20.10 t/ha). These results are almost similar with the findings of Khan et al. (2007) and Alam et al. (2008). Maximum shelf life was found in BARI Hybrid Patal-1 (15.0 days) followed by PG014×M 14.33 days), PG007× $\times \mathrm{M}_{2}$ (14 days) and PG017 $\times \mathrm{M}_{2}$ (12.50 days) while the lowest shelf life was found in PG005 $\times \mathrm{M}_{1}$ (6.23 days).

Table 3. Pulp weight, number of seeds per fruit, weight of seeds per fruit, duration of fruit harvest, fruit yield and shelf life of nineteen hybrid pointed gourd lines

\begin{tabular}{|c|c|c|c|c|c|c|}
\hline Hybrid lines & $\begin{array}{c}\text { Pulp } \\
\text { weight (g) }\end{array}$ & $\begin{array}{c}\text { Number } \\
\text { of } \\
\text { seeds/fruit }\end{array}$ & $\begin{array}{l}\text { Weight of } \\
\text { seeds/fruit } \\
\text { (g) }\end{array}$ & $\begin{array}{c}\text { Duration } \\
\text { of fruit } \\
\text { harvest } \\
\text { (days) }\end{array}$ & $\begin{array}{l}\text { Fruit } \\
\text { yield } \\
\text { (t/ha) }\end{array}$ & $\begin{array}{c}\text { Shelf life } \\
\text { (days) }\end{array}$ \\
\hline $\mathrm{PG} 002 \times \mathrm{M}_{1}$ & $34.00 \mathrm{~d}-\mathrm{h}$ & $22.33 b-d$ & $5.20 \mathrm{c}$ & $218 c-f$ & $28.84 \mathrm{~h}$ & $6.25 \mathrm{j}$ \\
\hline $\mathrm{PG} 003 \times \mathrm{M}_{1}$ & $38.00 \mathrm{c}$ & $22.66 b-d$ & $5.56 \mathrm{bc}$ & $219 b-e$ & $29.80 \mathrm{~h}$ & $7.25 \mathrm{ij}$ \\
\hline $\mathrm{PG} 004 \times \mathrm{M}_{1}$ & $38.10 \mathrm{c}$ & $22.67 b-d$ & $5.40 \mathrm{bc}$ & $214 \mathrm{gh}$ & $34.30 \mathrm{e}-\mathrm{g}$ & $11.23 \mathrm{c}-\mathrm{e}$ \\
\hline $\mathrm{PG} 005 \times \mathrm{M}_{1}$ & $33.00 \mathrm{e}-\mathrm{i}$ & $16.66 f$ & $4.13 \mathrm{~d}$ & 216d-g & $29.23 \mathrm{~h}$ & $6.23 \mathrm{j}$ \\
\hline $\mathrm{PG} 006 \times \mathrm{M}_{1}$ & $31.00 \mathrm{~g}-\mathrm{j}$ & $23.00 \mathrm{~b}-\mathrm{d}$ & $5.30 b c$ & $215 f g h$ & $35.04 \mathrm{~d}-\mathrm{f}$ & $10.50 \mathrm{~d}-\mathrm{f}$ \\
\hline $\mathrm{PG} 008 \times \mathrm{M}_{1}$ & $37.00 \mathrm{c}-\mathrm{e}$ & $22.23 b-d$ & $5.43 b c$ & $214 \mathrm{gh}$ & $29.20 \mathrm{~h}$ & $12.00 \mathrm{c}$ \\
\hline $\mathrm{PG} 012 \times \mathrm{M}_{1}$ & $29.00 \mathrm{ij}$ & $24.00 \mathrm{a}-\mathrm{d}$ & $4.16 \mathrm{~d}$ & $216 d-g$ & $35.06 \mathrm{~b}-\mathrm{f}$ & $9.66 \mathrm{fg}$ \\
\hline PG014×M 1 & $39.10 \mathrm{~b}-\mathrm{d}$ & $27.33 \mathrm{a}$ & $5.33 b c$ & 215 fgh & $35.58 \mathrm{c}-\mathrm{e}$ & $14.33 b$ \\
\hline $\mathrm{PG} 022 \times \mathrm{M}_{1}$ & $17.20 \mathrm{k}$ & $23.00 \mathrm{bc}$ & $5.20 \mathrm{c}$ & $213 \mathrm{gh}$ & $20.10 \mathrm{j}$ & $9.00 \mathrm{gh}$ \\
\hline $\mathrm{PG} 002 \times \mathrm{M}_{2}$ & $26.50 \mathrm{j}$ & $21.66 \mathrm{~cd}$ & $5.40 \mathrm{bc}$ & $212 \mathrm{~h}$ & $23.10 \mathrm{i}$ & 8.00hi \\
\hline $\mathrm{PG} 005 \times \mathrm{M}_{2}$ & $38.50 \mathrm{~b}-\mathrm{d}$ & $21.00 \mathrm{de}$ & $5.41 b c$ & $212 \mathrm{~h}$ & $34.03 \mathrm{e}-\mathrm{g}$ & $11.3 \mathrm{c}-\mathrm{e}$ \\
\hline $\mathrm{PG} 006 \times \mathrm{M}_{2}$ & $32.00 \mathrm{f}-\mathrm{i}$ & $18.00 \mathrm{ef}$ & $7.10 \mathrm{a}$ & $218 \mathrm{c}-\mathrm{f}$ & $33.01 \mathrm{~g}$ & $9.03 \mathrm{gh}$ \\
\hline $\mathrm{PG} 007 \times \mathrm{M}_{2}$ & $35.60 \mathrm{c}-\mathrm{g}$ & $25.33 \mathrm{ab}$ & $5.30 \mathrm{bc}$ & $220 b c$ & $36.85 \mathrm{c}$ & $14.00 \mathrm{~b}$ \\
\hline $\mathrm{PG} 008 \times \mathrm{M}_{2}$ & $42.20 \mathrm{ab}$ & $24.66 a-c$ & $5.20 \mathrm{c}$ & $219 b d$ & $39.23 b$ & $8.66 \mathrm{gh}$ \\
\hline $\mathrm{PG} 009 \times \mathrm{M}_{2}$ & $37.40 \mathrm{c}-\mathrm{e}$ & $20.66 \mathrm{de}$ & $5.56 b c$ & $222 \mathrm{ab}$ & $45.74 \mathrm{a}$ & $11.50 \mathrm{~cd}$ \\
\hline $\mathrm{PG} 017 \times \mathrm{M}_{2}$ & $36.20 c-f$ & $23.00 \mathrm{~b}-\mathrm{d}$ & $5.40 \mathrm{bc}$ & $223 a$ & $36.19 \mathrm{~cd}$ & $12.50 \mathrm{c}$ \\
\hline $\mathrm{PG} 018 \times \mathrm{M}_{2}$ & $39.50 \mathrm{bc}$ & $25.66 \mathrm{ab}$ & $7.20 \mathrm{a}$ & $216 \mathrm{efg}$ & $34.35 \mathrm{e}-\mathrm{g}$ & $9.36 \mathrm{~h}$ \\
\hline $\mathrm{PG} 025 \times \mathrm{M}_{2}$ & $35.00 \mathrm{c}-\mathrm{g}$ & $25.00 \mathrm{a}-\mathrm{c}$ & $5.73 b$ & $218 \mathrm{c}-\mathrm{f}$ & $33.81 \mathrm{fg}$ & $10.00 \mathrm{e}-\mathrm{g}$ \\
\hline BARI Hybrid Patal -1 & $44.20 \mathrm{a}$ & $23.00 \mathrm{~b}-\mathrm{d}$ & $5.40 \mathrm{bc}$ & $218 \mathrm{c}-\mathrm{f}$ & $35.94 \mathrm{~cd}$ & $15.00 \mathrm{a}$ \\
\hline $\mathrm{CV}(\%)$ & 7.32 & 8.14 & 4.94 & 4.58 & 2.50 & 7.32 \\
\hline
\end{tabular}

Means with uncommon letter (s) are significantly different at $5 \%$ level by DMRT. 


\section{D) Leaf colour, leaf tip and leaf margin}

The leaves of pointed gourd lines showed wide variation in different leaf characteristics (Table 4). The colour of leaves was light green, green and deep green. The leaves of the lines, PG017 $\times \mathrm{M}_{2}, \mathrm{PG} 018 \times \mathrm{M}_{2}$, and $\mathrm{PG} 025 \times \mathrm{M}_{2}$ were deep green while PG003 $\times \mathrm{M}_{1}, \mathrm{PG} 004 \times \mathrm{M}_{1}, \mathrm{PG} 005 \times \mathrm{M}_{1}, \mathrm{PG} 006 \times \mathrm{M}_{1}, \mathrm{PG} 008 \times \mathrm{M}_{1}$, PG014 $\times \mathrm{M}_{1}, \mathrm{PG} 005 \times \mathrm{M}_{2}$ and PG006 $\times \mathrm{M}_{2}$ were green. The rest of the lines had light green leaves. The two lines $\mathrm{PG} 005 \times \mathrm{M}_{1}$ and $\mathrm{PG} 018 \times \mathrm{M}_{2}$, and the variety BARI Hybrid Patal 1 produced blunt leaves, while the rest of the lines produced pointed leaves. The leaf margin of $\mathrm{PG} 002 \times \mathrm{M}_{1}$ and $\mathrm{PG} 009 \times \mathrm{M}_{2}$ were slightly serrated, while PG003 $\times \mathrm{M}_{1}, \mathrm{PG} 004 \times \mathrm{M}_{1}, \mathrm{PG} 012 \times \mathrm{M}_{1}, \mathrm{PG} 005 \times \mathrm{M}_{2}$ and PG017 $\times \mathrm{M}_{2}$ and PG004 $\times M_{1}$ were serrated. The leaf margin of the lines $P G 005 \times M_{1}$, $\mathrm{PG} 008 \times \mathrm{M}_{1}, \quad \mathrm{PG} 014 \times \mathrm{M}_{1}, \quad \mathrm{PG} 022 \times \mathrm{M}_{1}, \quad \mathrm{PG} 002 \times \mathrm{M}_{2}, \quad \mathrm{PG} 006 \times \mathrm{M}_{2}, \quad \mathrm{PG} 007 \times \mathrm{M}_{2}$, PG008 $\times \mathrm{M}_{2}$ were entire and the remaining had undulated margin. These results are in agreement with the findings of Khan et al. (2007) who reported to have variation in type of margin of pointed gourd.

Table 4. Leaf characteristics of nineteen hybrid pointed gourd lines

\begin{tabular}{ccccc}
\hline Hybrid lines & Leaf colour & Leaf tip & Leaf margin \\
\hline PG002 $\times M_{1}$ & Light green & Pointed & Slightly serrated \\
PG003 $\times M_{1}$ & Green & Pointed & Serrated \\
PG004 $\times M_{1}$ & Green & Pointed & Serrated \\
PG005 $\times M_{1}$ & Green & Blunt & Entire \\
PG006 $\times M_{1}$ & Green & Pointed & Undulated \\
PG008 $\times M_{1}$ & Green & Pointed & Entire \\
PG012 $\times M_{1}$ & Light Green & Pointed & Serrated \\
PG014 $\times M_{1}$ & Green & Pointed & Entire \\
PG022 $\times M_{1}$ & Light green & Pointed & Entire \\
PG002 $\times M_{2}$ & Light green & Pointed & Entire \\
PG005 $\times M_{2}$ & Green & Pointed & Serrated \\
PG006 $\times M_{2}$ & Green & Pointed & Entire \\
PG007 $\times M_{2}$ & Light green & Pointed & Entire \\
PG008 $\times M_{2}$ & Light green & Pointed & Entire \\
PG009 $\times M_{2}$ & Light green & Pointed & Slightly serrated \\
PG017 $\times M_{2}$ & Deep Green & Pointed & Serrated \\
PG018 $\times M_{2}$ & Deep Green & Blunt & Undulated \\
PG025 $\times M_{2}$ & Deep Green & Pointed & Undulated \\
BARI Hybrid Patal-1 & Light green & Blunt & Undulated \\
\hline
\end{tabular}


Table 5. Fruit characteristics of nineteen hybrid pointed gourd lines at marketable

\begin{tabular}{|c|c|c|c|c|}
\hline Hybrid lines & Fruit colour & Stripes of fruit & $\begin{array}{l}\text { Shape of } \\
\text { fruit }\end{array}$ & $\begin{array}{c}\text { Curvature of } \\
\text { fruit }\end{array}$ \\
\hline $\mathrm{PG} 002 \times \mathrm{M}_{1}$ & Dark green & Green white stripe & Spindle & Slightly curved \\
\hline $\mathrm{PG} 003 \times \mathrm{M}_{1}$ & Whitish & No stripe & Spindle & Curved \\
\hline $\mathrm{PG} 004 \times \mathrm{M}_{1}$ & Dark green & White stripe & Spindle & Curved \\
\hline $\mathrm{PG} 005 \times \mathrm{M}_{1}$ & Light green & No stripe & Cylindrical & Slightly curved \\
\hline $\mathrm{PG} 006 \times \mathrm{M}_{1}$ & light green & Light green stripe & Cylindrical & Straight \\
\hline $\mathrm{PG} 008 \times \mathrm{M}_{1}$ & Dark green & White stripe & Cylindrical & Slightly curved \\
\hline $\mathrm{PG} 012 \times \mathrm{M}_{1}$ & Green & White stripe & Oval & Straight \\
\hline $\mathrm{PG} 014 \times \mathrm{M}_{1}$ & Green & Green white stripe & Cylindrical & Straight \\
\hline $\mathrm{PG} 022 \times \mathrm{M}_{1}$ & Dark green & Green white stripe & Oval & Straight \\
\hline $\mathrm{PG} 002 \times \mathrm{M}_{2}$ & Light green & White stripe & Spindle & Curved \\
\hline $\mathrm{PG} 005 \times \mathrm{M}_{2}$ & Whitish & No stripe & Spindle & Curved \\
\hline $\mathrm{PG} 006 \times \mathrm{M}_{2}$ & Dark green & Green white stripe & Cylindrical & Straight \\
\hline $\mathrm{PG} 007 \times \mathrm{M}_{2}$ & Dark green & Green white stripe & Cylindrical & Slightly curved \\
\hline $\mathrm{PG} 008 \times \mathrm{M}_{2}$ & Dark green & Green white stripe & Cylindrical & Curved \\
\hline $\mathrm{PG} 009 \times \mathrm{M}_{2}$ & Dark green & Green white stripe & Spindle & Curved \\
\hline $\mathrm{PG} 017 \times \mathrm{M}_{2}$ & Dark green & Green white stripe & Oval & Straight \\
\hline $\mathrm{PG} 018 \times \mathrm{M}_{2}$ & Green & Green white stripe & Spindle & Slightly curved \\
\hline $\mathrm{PG} 025 \times \mathrm{M}_{2}$ & Light green & Green white stripe & Oval & Slightly curved \\
\hline BARI Hybrid Patal -1 & Dark green & Green white stripe & Cylindrical & Slightly curved \\
\hline
\end{tabular}

\section{E) Colour, shape, stripe and curvature of fruit}

The fruits of hybrid pointed gourd lines showed variation in colour, shape, stripes and curvature (Table 5). The colour of fruit was dark green in $\mathrm{PG} 002 \times \mathrm{M}_{1}$, $\mathrm{PG} 004 \times \mathrm{M}_{1}, \quad \mathrm{PG} 008 \times \mathrm{M}_{1}, \quad \mathrm{PG} 022 \times \mathrm{M}_{1}, \quad \mathrm{PG} 006 \times \mathrm{M}_{2}, \quad \mathrm{PG} 007 \times \mathrm{M}_{2}, \quad \mathrm{PG} 008 \times \mathrm{M}_{2}$, PG009 $\times \mathrm{M}_{2}, \mathrm{PG} 017 \times \mathrm{M}_{2}$, and BARI Hybrid Patal. The fruit colour of PG003 $\times \mathrm{M}_{1}$ and PG005 $\times \mathrm{M}_{2}$ was whitish; whereas, PG005 $\times \mathrm{M}_{1}, \mathrm{PG} 006 \times \mathrm{M}_{1}, \mathrm{PG} 002 \times \mathrm{M}_{2}$ and $\mathrm{PG} 025 \times \mathrm{M}_{2}$ was light green. Variation in colour of pointed gourd has also been observed by Ram (2001) and Khan et al. (2007). The fruits of PG004 $\times \mathrm{M}_{1}$, $\mathrm{PG} 008 \times \mathrm{M}_{1}, \mathrm{PG} 012 \times \mathrm{M}_{1}$ and $\mathrm{PG} 002 \times \mathrm{M}_{2}$ showed white stripes and PG006 $\times \mathrm{M}_{1}$ showed light green stripes. On the other hand, the fruits of $P G 003 \times M_{1}$, $\mathrm{PG} 005 \times \mathrm{M}_{1}$ and $\mathrm{PG} 005 \times \mathrm{M}_{2}$ had no stripe. The rest of the lines produced fruits with green white stripes. The lines $P G 005 \times M_{1}, P G 006 \times M_{1}, P G 008 \times M_{1}$, 
$\mathrm{PG} 014 \times \mathrm{M}_{1}, \quad \mathrm{PG} 006 \times \mathrm{M}_{2}, \quad \mathrm{PG} 007 \times \mathrm{M}_{2}, \quad \mathrm{PG} 008 \times \mathrm{M}_{2}$ and $\mathrm{PG} 027 \times \mathrm{M}_{2}$ produced cylindrical fruits, while $\mathrm{PG} 002 \times \mathrm{M}_{1}, \mathrm{PG} 003 \times \mathrm{M}_{1}, \mathrm{PG} 004 \times \mathrm{M}_{1}, \mathrm{PG} 002 \times \mathrm{M}_{2}$, $\mathrm{PG} 005 \times \mathrm{M}_{2}, \mathrm{PG} 009 \times \mathrm{M}_{2}$ and $\mathrm{PG} 018 \times \mathrm{M}_{2}$ produced spindle shaped fruits, but the rest of the lines produced oval shaped fruits. Variation in shape of pointed gourd was also reported by Khan et al. (2007). Slightly curved fruits were found in $\mathrm{PG} 002 \times \mathrm{M}_{1}, \mathrm{PG} 005 \times \mathrm{M}_{1}, \mathrm{PG} 008 \times \mathrm{M}_{1}, \mathrm{PG} 007 \times \mathrm{M}_{2}, \mathrm{PG} 018 \times \mathrm{M}_{2}, \mathrm{PG} 025 \times \mathrm{M}_{2}$ and BARi Hybrid Patal 1. On the contrary, curved fruits were produced by $\mathrm{PG} 003 \times \mathrm{M}_{1}, \mathrm{PG} 004 \times \mathrm{M}_{1}, \mathrm{PG} 002 \times \mathrm{M}_{2}, \mathrm{PG} 005 \times \mathrm{M}_{2}, \mathrm{PG} 008 \times \mathrm{M}_{2}$ and $\mathrm{PG} 009 \times \mathrm{M}_{2}$ but the rest produced straight fruits. The results are in conformity with the report of Prasad and Singh (1990).

\section{Conclusion}

Based on the above result, it can be concluded that the hybrid pointed gourd lines PG014 $\times \mathrm{M}_{1}, \mathrm{PG} 007 \times \mathrm{M}_{2}, \mathrm{PG} 008 \times \mathrm{M}_{2}, \mathrm{PG} 009 \times \mathrm{M}_{2}$ and $\mathrm{PG} 017 \times \mathrm{M}_{2}$ performed better in respect of yield and yield attributes. These five hybrid lines should be subjected for further evaluation to release as hybrid variety (ies).

\section{References}

Alam, M. A., M. G. Rabbani, E. H. M. S. Rahman, M. R. Kabir and M. S. N. Mandal. 2008. Evaluation of some collected pointed gourd genotypes and their relationship. Int. J. BioRes. 4(1):17-23.

Anonymous. 2016. Year Book of Agricultural Statististics-2015, Bangladesh Bureau of Statistics (BBS). Ministry of Planning, Govt. of the Peoples Republic of Bangladesh. P. 245.

Awal, A., M. S. M. J. Alam, M. R. Al, M. N. Hasan, S. R. Basunia and S. M. M. Rahman. 2005. In Vitro propagation of pointed gourd (Trichosanthes dioica Roxb.) from shoot tips. Biotec. 4(3): 221-224.

BARC. 2005. Fertilizer Recommendation Guide. Bangladesh Agril. Res. Council, Farmgate, Dhaka-1215. $191 \mathrm{P}$.

FAO. 1981. Food and Agricultural Organization of the United Nations. Soil Survey Project of Bangladesh. Soil Res. Tech. Pep. Pp. 101-159.

IBPGR. 1983. Descriptor for Pointed Gourd (Trichosanthes dioica Roxb.). International Board for Plant Genetic Resources. Rom, Italy.

Kabir, M. E. 2007. Genetic variability, correlation and path analysis of pointed gourd (Trichosanthes dioica Roxb.). M. S. Thesis. Dept. of Hort. and Post Harvest Tech., Sher-e-Bangla Agril Univ. Dhaka-1207. 78 P.

Khan, A. S. M. M. R., M. G. Rabbani, M. A. Siddique, and M. A. Islam. 2007. Characterization and evaluation of pointed gourd germplasm. Bangladesh J. Agril. Res. 32(1): 117-134.

Kumar, R, V. S. Brahmachari and R. Kumar 1995. Varietal assessment of Parwal (Trichosanthes dioica). Indian. J. Hort. 8(2): 165-168. 
Prasad, V. S. R. K. and D. P. Singh. 1990. Studies of morphological component of pointed gourd (Trichosanthes dioica). Indian. J. Hort. 47(3): 537-540.

Ram, D. 2001. Non-hierarchical Euclidean cluster analysis in pointed gourd. Indian J. Hort. 58(3): 264-268.

Rashid, M. M. 1993. Vegetable Science (in Bengali) $1^{\text {st }}$ ed. Bangla Academy, Dhaka. Bangladesh. Pp. 333-336.

Shanmugavelu, K G. 1989. Production Technology of Vegetable Crops. Oxford and IBH Pub. Co., New Delhi, India. Pp. 821-825.

Singh, A. K., R. D. Singh and K. Singh. 1992. Genetic Variability, heritability and genetic advance for some traits in pointed gourd (Trichasanthes dioica Roxb.). Haryana J. Hort. Sci. 21(3-4):236-240.

Singh, D. P. and V. S. R. K. Prasad. 1989. Variability and correlation studies in pointed gourd (Trichosanthes dioica Roxb). Indian J. Hor. 46(2): 204-209.

Singh, K. 1989. Pointed gourd (Trichosanthes dioica Roxb.). Indian Hort. 33: 35-38.

Singh, R. R., G. M. Mishra and R. N. Jha. 1985. Studies on varieties and scopes for improvement in pointed gourd (Thicosanthes dioica). South Indian Hort. 33(4): 257260. 
